\title{
Conhecimento popular e utilização dos medicamentos genéricos na população do município de Tubarão, SC
}

\author{
General awareness and use of generic medication \\ among citizens of Tubarão, state of Santa Catarina, Brazil
}

Carine Raquel Blatt ${ }^{1}$

Silvana Cristina Trauthman ${ }^{1}$

Edegar Henrique Schmidt ${ }^{1}$

Samuel Marchesan ${ }^{1}$

Luana May da Silva ${ }^{1}$

João Luiz Martins ${ }^{1}$

${ }^{1}$ Curso de Farmácia, Universidade do Sul de Santa Catarina. Campus Tubarão. Avenida José Acácio Moreira 787, Dehon. 88704-900 Tubarão SC

carine.blatt@gmail.com

\begin{abstract}
Although generic medication has been introduced in the country to offer an accessible alternative to brand-name medication, it represents only $14 \%$ of sales in number of units within the pharmaceutical market. The aim of this work was to research the level of awareness and the use of generic products among residents of the municipality of Tubarão, State of Santa Catarina, Brazil. A transversal study was carried out with a sample of 234 interviewees, distributed among municipal areas. With regard to use, the majority of those interviewed had used generic medication, and half of them had at least one such product in their home. To verify awareness of different types of medication, pictures with the gener$i c$, brand name and similar packaging for paracetamol and atenolol were shown and 91\% were able to identify all products correctly. To be of higher economic standing, already having used generic products, believing that the generic medication has the same effect as the brand name medication, finding generic products in drugstores easily and being accustomed to buy generic products, were factors that were positively associated with the correct identification.
\end{abstract}

Key words Generic medication, General awareness, Use of medication
Resumo Embora os genéricos tenham sido introduzidos no país para oferecer alternativa mais acessivel aos medicamentos de referência, representam apenas $14 \%$ das vendas em unidades no conjunto do mercado farmacêutico. O objetivo deste trabalho foi pesquisar o nível de conhecimento e o grau de utilização de genéricos em residentes do município de Tubarão, SC. Para isso, realizou-se um estudo transversal com uma amostra de 234 entrevistados, estratificada por bairro. Quanto ao grau de utilização, a maioria dos entrevistados já havia utilizado genéricos, e metade possuía pelo menos um exemplar dessa opção em casa. Para verificar o conhecimento sobre os diferentes tipos de medicamentos, realizou-se um teste de identificação de figuras de embalagens representativas das versões genérico, de referência e similar do paracetamol e do atenolol, 91,0\% dos sujeitos identificaram corretamente todas as figuras. Ser de classe econômica mais elevada, já ter utilizado medicamento genérico, acreditar que o genérico tem o mesmo efeito que o medicamento de referência, encontrar medicamentos genéricos nas farmácias com facilidade e costumar comprar o genérico foram fatores associados positivamente com a identificação correta.

Palavras-chave Medicamento genérico, Conhecimento popular, Uso de medicamentos 


\section{Introdução}

Cerca de um terço da população mundial tem dificuldade de acesso aos medicamentos, o que decorre, principalmente, dos elevados custos e da dificuldade de regulação do mercado farmacêutico ${ }^{1}$. Por esses motivos, medicamentos genéricos são utilizados em vários países como agentes reguladores desse mercado, devido ao seu poder de influência na oferta e na demanda ${ }^{2-6}$.

No mesmo sentido, o Brasil tem empreendido importantes esforços para melhorar o acesso aos medicamentos a partir da promoção de políticas de saúde. Um deles foi a introdução dos medicamentos genéricos ${ }^{7,8}$, em 1999, com a publicação da Lei n. $9.787^{9}$, que estabelece esse tipo de medicamento e regulamenta a utilização de nomes genéricos em produtos farmacêuticos, além do registro, da produção, da prescrição e da venda desses insumos.

Apesar dessa política não ter proporcionado um aumento significativo no acesso aos genéricos, os consumidores passaram a ter a oportunidade de comprar medicamentos a preços mais acessíveis e com garantia de qualidade e intercambiamento $^{10}$.

Com a entrada dos medicamentos genéricos no mercado nacional, aumentou a concorrência entre os produtos. O genérico passou a conquistar o mercado, enquanto o de referência passou a competir fortemente para manter sua meta de vendas. Os preços reduzidos dos medicamentos genéricos são justificados pelo menor investimento em marketing e pela ausência de despesas com o desenvolvimento de princípios ativos e ensaios clínicos requeridos para um produto inovador ${ }^{5,10,11}$. No Brasil, a implantação dos medicamentos genéricos proporcionou uma redução média dos custos dos medicamentos, compreendida entre 40 e $62 \%$, segundo os valores encontrados por diferentes estudos ${ }^{3,12,13}$. A Associação Brasileira das Indústrias de Medicamentos Genéricos informa que os genéricos são 35\%, mais baratos que os de referência ${ }^{14}$.

Até junho de 2008, apesar de todas as estratégias de estímulo para o uso dos genéricos no Brasil, eles respondiam por apenas, $16,6 \%$ das vendas em unidades, no conjunto do mercado farmacêutico. Como comparação, nos EUA, mercado onde os genéricos já têm 20 anos de existência, o índice em volume de participação é de aproximadamente $60 \%{ }^{14}$.

Os fatores de resistência a sua utilização são a baixa disponibilidade desses produtos na farmácia $^{12}$, a falta de conhecimento dos consumi- dores ${ }^{15-20}$ o baixo estímulo à prescrição ${ }^{15,21}$, a falta de conhecimento dos médicos ${ }^{22}$ e dos farmacêu$\operatorname{ticos}^{23}$ e a falta de orientação para o uso ${ }^{24}$.

O objetivo deste estudo foi fazer um levantamento do conhecimento popular a respeito dos genéricos, bem como compreender como esse conhecimento influi na utilização deles.

\section{Metodologia}

O presente trabalho trata-se de um estudo transversal realizado no município de Tubarão, Santa Catarina, o qual possui aproximadamente 95.341 habitantes e está localizado na região sul do Estado ${ }^{25}$. Considerando a população total e o nível de prevalência de desinformação sobre genéricos de $46 \%$, resultado da pesquisa realizada pelo Ministério da Saúde ${ }^{15}$, o tamanho da amostra calculado para um índice de confiabilidade de 95\% foi de 195 indivíduos.

A amostra foi estratificada por bairro, segundo a metodologia descrita por Faria e Tavares Neto $^{19}$. Inicialmente, verificou-se o número de bairros do município, que perfazem um total de 26, segundo dados fornecidos pela prefeitura municipal. De cada bairro foi sorteada uma rua. A coleta de dados teve início pela casa com menor número, seguindo a ordem crescente até completar o número de 9 entrevistas por bairro, totalizando 234 entrevistas. Em cada casa, uma pessoa foi convidada a participar da pesquisa. Não havendo ninguém na casa ou alguém que preenchesse os critérios de inclusão, passava-se à casa de número imediatamente superior.

Aplicou-se um questionário estruturado, com perguntas abertas e fechadas, objetivando levantar dados socioeconômicos e informações sobre o conhecimento e a utilização de medicamentos.

Os critérios de inclusão foram pessoas com idade superior a 18 anos e que concordassem em assinar o termo de consentimento livre e esclarecido. Realizaram-se as entrevistas no período de julho a setembro de 2007.

Os fatores avaliados foram a utilização de medicamentos de uso contínuo, a quantidade de medicamentos comprados mensalmente, a fonte de obtenção, a opinião sobre a divulgação dos genéricos, a utilização prévia de genéricos, se acredita que tem o mesmo efeito que o de referência, se faz ou já fez comparação de preço entre o genérico e o de referência, se havia medicamentos genéricos em casa, se consegue identificar os medicamentos por meio de figuras, se os médicos que costuma consultar prescrevem genéricos, se 
encontra esse tipo de medicamento nas farmácias com facilidade, se costuma comprar e se aceitaria a troca efetuada pelo farmacêutico do medicamento de referência pelo genérico.

Para verificar o conhecimento dos entrevistados sobre os medicamentos, foram apresentadas três ilustrações de dois medicamentos (paracetamol e atenolol). Cada medicamento possuía uma ilustração que correspondia ao medicamento de referência, genérico e similar. Considerou-se como identificação correta aquele entrevistado que identificasse corretamente as seis ilustrações.

O paracetamol e o atenolol foram selecionados como amostras; o primeiro por ser um medicamento de uso comum, que poderia ser identificado mais facilmente pela população em geral; e o segundo, por de ser um medicamento usado cronicamente e não ter uso tão amplo como o primeiro. Dessa maneira, a identificação correta do primeiro poderia estar associada à experiência de uso prévio, mas a do segundo provavelmente, não. Os autores consideraram que, se o entrevistado acertasse as seis figuras, ele realmente conheceria a definição e os conceitos que caracterizam os medicamentos genérico, de referência e similar.

Para a identificação da classe econômica dos entrevistados, foi utilizado o Critério de Classificação Econômica Brasil. Esse questionário pa- dronizado tem como função estimar o poder de compra das pessoas e das famílias urbanas ${ }^{26}$.

As variáveis contínuas foram analisadas por meio de medidas de tendência central, e as variáveis categóricas, através da distribuição de frequências. As associações entre as variáveis preditoras e a identificação correta das figuras de medicamentos genéricos foram testadas mediante a razão de prevalência. Nas análises, o resultado considerado como de significância estatística foi aquele que apresentou probabilidade de erro do tipo I igual ou inferior a $5 \%(\mathrm{p} \leq 0,05)$.

O trabalho foi submetido e aprovado pelo Comitê de Ética da Universidade do Sul de Santa Catarina.

\section{Resultados}

O estudo incluiu 234 indivíduos residentes nos 26 bairros do município de Tubarão, SC. Não se registraram o número de casas que não tinham nenhum morador durante a visita do entrevistador e o número de indivíduos que se recusaram a participar do estudo.

As características do perfil socioeconômico e de consumo de medicamentos podem ser observadas na Tabela 1. Entre os indivíduos entrevistados percebeu-se o predomínio do sexo femini-

Tabela 1. Perfil socioeconômico e consumo dos medicamentos de residentes do município de Tubarão, SC.

\begin{tabular}{|c|c|}
\hline Características & Valores $\%(n=234)$ \\
\hline \multicolumn{2}{|l|}{ Idade } \\
\hline Intervalo (18 e 84 anos) & Média $=45,9 \mathrm{DP}= \pm 17,0$ \\
\hline \multicolumn{2}{|l|}{ Sexo } \\
\hline Feminino & 75,6 \\
\hline \multicolumn{2}{|l|}{ Escolaridade } \\
\hline Intervalo ( 0 e 23 anos) & Média $=8,2 \mathrm{DP}= \pm 3,6$ \\
\hline \multicolumn{2}{|l|}{ Classe econômica } \\
\hline A2 & 0,9 \\
\hline B1 & 6,0 \\
\hline B2 & 19,2 \\
\hline $\mathrm{C}$ & 47,4 \\
\hline $\mathrm{D}$ & 21,4 \\
\hline $\mathrm{E}$ & 5,1 \\
\hline \multicolumn{2}{|l|}{ Utiliza medicamentos de uso contínuo } \\
\hline Sim & 43,6 \\
\hline \multicolumn{2}{|c|}{ Número de medicamentos comprados por mês para seu consumo } \\
\hline Nenhum & 22,6 \\
\hline 1 a 3 & 59,0 \\
\hline 4 a 7 & 17,1 \\
\hline Mais de 7 & 1,3 \\
\hline \multicolumn{2}{|c|}{ Recebe medicamentos nas unidades de saúde do município } \\
\hline Sim & 26,5 \\
\hline
\end{tabular}


no e da classe C. A média de idade foi de 45,9 anos, e a escolaridade, de 8,2 anos.

A respeito de como os entrevistados identificavam os medicamentos genéricos, a letra " $G$ ", a "tarja amarela" e a frase "Medicamento Genérico" na faixa foram as principais formas de identificação do medicamento genérico, com percentuais de resposta de 37,6, 28,2 e 23,9 respectivamente.

Como pode ser visualizado na Tabela 2 , ao apresentarem as figuras, um total de $91,0 \%$ dos entrevistados identificaram corretamente os dois exemplos de genéricos apresentados. Contudo, apenas $25,6 \%$ identificaram corretamente as seis figuras.

Aos entrevistados que afirmavam possuir algum medicamento genérico em casa, solicitouse a caixa para conferência. Houve correspondência entre o produto apresentado e a embalagem do medicamento que acreditavam ser um produto genérico em $75,6 \%$ das vezes.

Os medicamentos genéricos encontrados com maior frequência na casa dos entrevistados foram paracetamol $(21,9 \%)$, amoxicilina $(10,4 \%)$, dipirona $(5,2 \%)$, fluoxetina $(4,2 \%)$, diclofenaco $(4,2 \%)$ e atenolol $(4,2 \%)$.

Na Tabela 3 apresentam-se os dados relacionados ao acesso, informação e divulgação dos medicamentos genéricos. Conforme relatos dos pacientes, cerca de 35\% dos médicos que costumam consultar nunca prescrevem genéricos. Aproximadamente $70 \%$ dos entrevistados relataram encontrar os medicamentos genéricos nas farmácias com facilidade. As principais fontes de informação citadas sobre os medicamentos genéricos foram a televisão e a farmácia.

Cerca de $85 \%$ dos entrevistados já utilizaram medicamentos genéricos, dos quais $77,8 \%$ costumam comprar com frequência. Em aproximadamente $69,2 \%$ dos casos, o próprio entrevistado relata ser o responsável pela solicitação. Também foram citados a sugestão do farmacêutico $(37,4 \%)$, a prescrição médica $(19,8 \%)$ e o fornecimento do SUS $(1,1 \%)$.

A maioria dos entrevistados já realizou comparação do preço entre o genérico e o de referên- cia, tendo sido o primeiro mais barato que o segundo em $97 \%$ das respostas afirmativas para a comparação de preço.

Em relação à confiança dos entrevistados nos medicamentos genéricos, $76,9 \%$ acreditam que estes têm o mesmo efeito que os de marca. Quase $90 \%$ dos que utilizaram os genéricos afirmaram ter ficado satisfeitos com o resultado.

Quanto ao poder de escolha entre o medicamento de referência e o genérico, $74,4 \%$ dos entrevistados relatam que escolheriam o genérico. Os principais motivos citados foram já ter utilizado (99,6\%), ter comparado com os preços dos medicamentos de referência $(67,9 \%)$ e acreditar em igual efeito terapêutico (50,4\%). Além disso, $73,1 \%$ dos entrevistados aceitavam a troca efetuada pelo farmacêutico do medicamento de referência pelo genérico.

Na Tabela 4 apresentam-se a razão de prevalência com o intervalo de confiança de $95 \%$ e a associação entre as variáveis independentes e a identificação correta das duas ilustrações de medicamentos genéricos mostradas aos entrevistados. A associação foi estatisticamente significativa somente com o fato de pertencer às classes $A$, $\mathrm{B}$ ou $\mathrm{C}$, já ter utilizado medicamento genérico, acreditar que o genérico tem o mesmo efeito que o medicamento de referência, encontrar medicamentos genéricos nas farmácias com facilidade e costumar comprar o genérico.

\section{Discussão}

A maioria dos pesquisados $(91,0 \%)$ identificou corretamente a figura do medicamento genérico entre os demais para os dois exemplos apresentados. Dois estudos anteriores realizados nesse mesmo município apresentam valores inferiores aos achados atuais. No estudo realizado em 2002 por acadêmicos de Farmácia da Universidade do Sul de Santa Catarina na população de Tubarão, $58 \%$ relatavam conhecer medicamento genérico, no entanto apenas $7 \%$ sabiam diferenciá-lo ${ }^{17}$. Em estudo realizado em 2005, também por acadêmi-

Tabela 2. Identificação correta dos diferentes tipos de medicamentos através das figuras por residentes do município de Tubarão, SC.

\begin{tabular}{lcccc}
\hline & Genérico & Referência & Similar & Todos \\
\hline Atenolol & $91,1 \%$ & $32,5 \%$ & $34,2 \%$ & $31,6 \%$ \\
Paracetamol & $91,9 \%$ & $44,4 \%$ & $44,9 \%$ & $42,3 \%$ \\
Atenolol + paracetamol & $91,0 \%$ & $26,1 \%$ & $27,4 \%$ & $26,1 \%$ \\
\hline
\end{tabular}


Tabela 3. Dados relacionados ao acesso, informação e divulgação dos medicamentos genéricos por residentes do município de Tubarão, SC.

\begin{tabular}{lc}
\hline \multicolumn{1}{c}{ Características } & Valores $\%(\mathbf{n}=\mathbf{2 3 4})$ \\
\hline Frequência de prescrição dos medicamentos genéricos & 23,5 \\
Sempre & 26,5 \\
Às vezes & 14,5 \\
Raramente & 34,6 \\
Nunca & 0,9 \\
Não sabe informar & \\
Facilidade em encontrar medicamentos genéricos nas farmácias & 69,2 \\
Sempre & 18.8 \\
Às vezes & 9,0 \\
Raramente & 3,0 \\
Nunca & \\
Opinião sobre a divulgação dos medicamentos genéricos & 7,3 \\
Ótima & 30,3 \\
Boa & 57,7 \\
Regular & 4,3 \\
Ruim & \\
Fonte de informações sobre os medicamentos genéricos & \\
Televisão & 72,6 \\
Farmácia & 33,3 \\
Médico & 12,8 \\
Rádio & 12,8 \\
Farmacêutico & 9,4 \\
Vizinhos & 5,6 \\
Jornal & 4,3 \\
Faculdade & 0,4 \\
Propaganda de rua & 0,4 \\
Internet & 1,7 \\
Nunca obteve informação & 0,4 \\
Prefere não responder & 0,4 \\
\hline Fon & \\
\hline
\end{tabular}

* Foi permitido mencionar mais de uma informação.

cos da mesma instituição, com uma população de 209 indivíduos pertencentes a grupos de idosos, $86 \%$ identificaram corretamente as embalagens de medicamentos genéricos ${ }^{18}$. Esses dados mostram que, ao longo dos anos a partir da vigência da Lei dos Genéricos, a população vem melhorando seus conhecimentos e ampliando suas experiências quanto aos genéricos.

Comparativamente, em estudo realizado no Recife, $68,1 \%$ dos usuários do serviço público identificaram e diferenciaram corretamente os genéricos ${ }^{20}$. No estudo realizado no Sul do Brasil, o percentual de pacientes que diferenciou corretamente a figura do medicamento genérico em relação ao de referência foi de $52 \%{ }^{16}$. No Acre, o percentual foi de $22 \%{ }^{19}$. Na pesquisa da Anvisa, realizada com uma amostra de 2.200 indivíduos em 236 municípios, esse percentual foi de $71 \%{ }^{15}$.

Diante do questionamento referente ao elemento pelo qual ocorre a identificação do medi- camento genérico, os entrevistados citaram a letra "G" como o item mais lembrado, seguido da tarja amarela e da frase "Medicamento Genérico". Esses elementos já foram citados como os principais meios de identificação em outras pesquisas realizadas no Brasil ${ }^{15,18}$ e mostram a importância da legislação adotada no país, que obriga a presença da tarja amarela com a grafia do medicamento genérico e da letra $\mathrm{G}$ em destaque na embalagem externa. Essa identidade visual diferenciada facilita a identificação pelo consumidor e a distinção entre o genérico e o de marca.

Com base nos resultados aqui citados, podese afirmar que a população deste estudo é capaz de identificar os medicamentos genéricos. Essa constatação foi reforçada quando foi solicitado ao entrevistado que apresentasse uma embalagem de medicamento genérico que houvesse no domicílio, tendo havido uma confirmação para $75,6 \%$ das especialidades farmacêuticas. 
Tabela 4. Razão de prevalência e intervalo de confiança de 95\% para a associação entre as variáveis independentes e a correta identificação das ilustrações dos medicamentos genéricos por residentes do município de Tubarão, SC.

\begin{tabular}{|c|c|c|c|c|}
\hline \multirow[b]{2}{*}{ Variável preditora } & \multicolumn{2}{|c|}{$\begin{array}{l}\text { Identificação correta } \\
\text { através das figuras } \\
\text { dos medicamentos }\end{array}$} & \multirow{2}{*}{$\begin{array}{l}\text { Razão de prevalência } \\
\text { (IC 95\%) }\end{array}$} & \multirow[b]{2}{*}{$p$} \\
\hline & Sim & Não & & \\
\hline Sexo & & & $1,02(0,93-1,13)$ & 0,6373 \\
\hline Feminino & 162 & 15 & & \\
\hline Masculino & 51 & 6 & & \\
\hline Escolaridade & & & $0,99(0,91-1,09)$ & 0,8953 \\
\hline$>8$ anos & 58 & 6 & & \\
\hline$\leq 8$ anos & 155 & 15 & & \\
\hline Classe econômica & & & $1,21(1,06-1,39)$ & 0,0000 \\
\hline $\mathrm{A}, \mathrm{B}, \mathrm{C}$ & 165 & 8 & & \\
\hline $\mathrm{D}, \mathrm{E}$ & 48 & 13 & & \\
\hline Utiliza medicamentos de uso contínuo & & & $0,97(0,89-1,05)$ & 0,3944 \\
\hline Sim & 91 & 111 & & \\
\hline Não & 122 & 10 & & \\
\hline Quantidade de medicamentos comprados mensalmente & & & $0,93(0,82-1,06)$ & 0,2060 \\
\hline Quatro ou mais & 37 & 6 & & \\
\hline Menos que quatro & 176 & 15 & & \\
\hline Adquire os medicamentos no posto de saúde & & & $0,92(0,83-1,03)$ & 0,0947 \\
\hline Sim & 55 & 9 & & \\
\hline Não & 158 & 12 & & \\
\hline Considera como boa a divulgação dos genéricos no Brasil & & & $1,00(0,92-1,09)$ & 0,9952 \\
\hline Sim & 132 & 13 & & \\
\hline Não & 81 & 8 & & \\
\hline Já utilizou medicamentos genéricos & & & $1,45(1,14-1,85)$ & 0,0000 \\
\hline Sim & 190 & 9 & & \\
\hline Não & 23 & 12 & & \\
\hline Acredita que o medicamento genérico tem o mesmo efeito & & & $1,12(0,99-1,27)$ & 0,0241 \\
\hline Sim & 168 & 12 & & \\
\hline Não & 45 & 9 & & \\
\hline Escolheria o genérico em detrimento do medicamento de & & & & \\
\hline referência & & & $1,07(0,96-1,19)$ & 0,1707 \\
\hline Sim & 161 & 13 & & \\
\hline Não & 52 & 8 & & \\
\hline Já fez comparação de preço entre genérico e referência & & & $1,10(0,95-1,28)$ & 0,0930 \\
\hline Sim & 182 & 15 & & \\
\hline Não & 31 & 6 & & \\
\hline Os médicos que você costuma consultar prescrevem & & & & \\
\hline medicamentos genéricos sempre & & & $1,02(0,94-1,12)$ & 0,6137 \\
\hline Sim & 51 & 4 & & \\
\hline Não & 162 & 17 & & \\
\hline Encontra medicamentos genéricos nas farmácias com facilidades & & & $1,16(1,040-1,30)$ & 0,0012 \\
\hline Sim & 154 & 8 & & \\
\hline Não & 59 & 13 & & \\
\hline Costuma comprar medicamentos genéricos & & & $1,16(1,01-1,33)$ & 0,0033 \\
\hline Sim & 171 & 11 & & \\
\hline Não & 42 & 10 & & \\
\hline Aceita a troca por genéricos pelo farmacêutico & & & $1,06(0,95-1,17)$ & 0,2264 \\
\hline Sim & 158 & 13 & & \\
\hline Não & 55 & 8 & & \\
\hline Possui medicamento genérico em casa & & & $1,04(0,96-1,13)$ & 0,2897 \\
\hline Sim & 117 & 9 & & \\
\hline Não & 96 & 12 & & \\
\hline
\end{tabular}


Contudo, considerando que apenas 25,6\% dos entrevistados identificaram corretamente as seis figuras, verificam-se ainda dificuldades na identificação do medicamento de referência e similar. Muitos entrevistados não conseguiram diferenciar estes medicamentos.

Tal dificuldade já foi constatada em outra pesquisa, realizada em 2004 pela Associação das Indústrias de Medicamentos Genéricos, com amostra de 900 consumidores de quatro capitais brasileiras, em que 30\% dos consumidores desconheciam o que era medicamento similar, e $78 \%$ o que era medicamento de referência ${ }^{27}$. Nesse caso, fica evidente a importância do fornecimento de informações adicionais durante a prescrição e a dispensação dos medicamentos.

Entre as variáveis pesquisadas, o fato de pertencer às classes $\mathrm{A}, \mathrm{B}$ ou $\mathrm{C}$, já ter utilizado medicamento genérico, acreditar que o genérico tem o mesmo efeito que o medicamento de referência, encontrar medicamentos genéricos nas farmácias com facilidade e costumar comprar o genérico foram estatisticamente significativos para a associação com a identificação correta dos medicamentos genéricos. Em outro estudo, o conhecimento sobre medicamentos genéricos foi de $22,1 \%$, o qual predominou também em pessoas com maiores indicadores socioeconômicos ${ }^{19}$. No estudo de Rocha et al. ${ }^{20}$, a escolaridade e a idade também foram associadas significativamente com tal conhecimento. Considerando que a escolaridade é uma variável que influencia o entendimento e a compreensão do genérico, tais achados mostram a importância das campanhas de divulgação, principalmente para a população de baixa escolaridade, e de esclarecimento sobre a identificação dos genéricos.

A maioria dos entrevistados já os utilizou pelo menos uma vez, e a metade possuía genéricos em casa, especialmente analgésicos, anti-hipertensivos, antibióticos e antidepressivos.

Dos entrevistados, $85 \%$ relataram ter utilizado alguma vez medicamento genérico, tendo a maioria aprovado o produto, e 76,9\% dos indivíduos acreditam que o medicamento genérico tem o mesmo efeito que o de marca. Apesar dos achados, os autores Hernandes e Júnior ${ }^{28}$ afirmam que diversos estudos já realizados no Brasil divergem quanto à aceitação do medicamento genérico e apontam para indícios de que o consumidor tem insegurança em relação ao efeito desse tipo de medicamento.

Segundo Rumel et al. ${ }^{29}$, a variabilidade biológica entre humanos e a não-similaridade absoluta entre medicamentos da mesma classe terapêu- tica ou mesmo genéricos levam, em alguns casos, a problemas de intercambialidade. Esses fatores são considerados como influenciadores na prescrição dos medicamentos genéricos e podem influenciar também na opinião da população leiga.

Nesta pesquisa, os principais motivos citados pela escolha do genérico em detrimento ao de marca foram já ter utilizado o medicamento genérico e ser mais barato que o de marca, mostrando o grande impacto do preço na escolha do medicamento, o que já havia sido apontado em outros estudos 6,15 .

Entre os entrevistados, 34,6\% responderam que os médicos que costumam consultar nunca prescrevem genéricos, e apenas 23,5\% que sempre prescrevem. Esses achados assemelham-se aos obtidos em outra pesquisa, em que apenas $22 \%$ das prescrições haviam sido feitas pela nomenclatura genérica da Anvisa ${ }^{15}$. Tal fato mostra a perspectiva do usuário e o número baixo de prescrições pelo nome genérico.

$\mathrm{Na}$ pesquisa realizada pela Anvisa ${ }^{15}$, na época da implantação dos genéricos, $80 \%$ das receitas avaliadas eram de medicamentos de marca, ainda que $78 \%$ dos médicos se manifestassem de forma positiva sobre a qualidade dos genéricos. Estudos demonstram que uma boa medida para aumentar a prescrição de genéricos é ampliar o nível de conhecimento dos prescritores sobre estes ${ }^{5,22,24}$.

Os medicamentos de referência e similar têm seu processo de difusão muito mais dinâmico que os genéricos devido aos instrumentos de persuasão dos laboratórios, entre os quais a figura do representante é a principal. É ele quem fornece ao médico as informações básicas para formar o conhecimento que o induzirá a receitar o medicamento ${ }^{7,30}$. Isso não significa que os genéricos não sejam propagandeados, mas sim que isso ocorre com menor frequência em relação aos medicamentos similares e aos de referência.

O farmacêutico foi citado como o responsável por ofertar medicamentos genéricos durante a aquisição de medicamentos. Embora farmacêuticos, balconistas e proprietários tenham consciência positiva em relação ao medicamento genérico, muitos dispensam medicamentos similares, devido a "bonificações" sobre a venda, especialmente dos similares ${ }^{31}$.

Entre a maioria dos entrevistados, a opinião sobre a divulgação de medicamentos genéricos no Brasil é considerada como boa. A televisão foi considerada o principal veículo de comunicação que proporcionou orientação e esclarecimentos a respeito de medicamentos genéricos. Numa pesquisa realizada em Pernambuco, foi conside- 
rado que os meios de comunicação de massa, em especial a televisão, constituem veículo adequado de comunicação sobre os medicamentos genéricos ${ }^{20}$. Portanto, pode-se atingir consumidores por meio de programas de educação vinculados pela mídia de massa. Este também foi o principal veículo de informação identificado por um estudo na Espanha para a divulgação dos genéricos naquele país ${ }^{30}$.

No Brasil, conforme apontam Dias e Romano-Lieber ${ }^{10}$, foi o respaldo da mídia que proporcionou ao governo o apoio popular. Nesse estudo, além da televisão, o segundo responsável apontado por prover informações foram os estabelecimentos farmacêuticos $(26,5 \%)$, seguidos do profissional farmacêutico $(9,1 \%)$. Ainda como o responsável por apresentar informações sobre medicamentos genéricos e o médico foi mencionado por $8,3 \%$. Tais números indicam a perspectiva do usuário, e a pouca informação fornecida aos pacientes pelos prescritores e dispensadores.

Quanto à substituição de medicamento realizado pelo farmacêutico, a troca do medicamento de referência pelo genérico seria bem aceita por $76,5 \%$ dos entrevistados, manifestando, dessa forma, confiança e aceitação, que parecem ser motivadas muito mais pela experiência de uso do que pelo acesso a informações confiáveis.

\section{Conclusão}

A população deste estudo mostrou ter um bom nível de conhecimento, o bastante para identificar os medicamentos genéricos, e aceita bem o uso destes. Além disso, mostrou preferência pelo genérico, estando satisfeita com os resultados obtidos no emprego e no custo menor, comparado ao medicamento de referência. Contudo, ainda apresentou dificuldades em diferenciar o de referência e o similar.

Na prática, não existe nada que possa ser utilizado para diferenciar medicamento de referência do similar, somente a informação fornecida oralmente no momento da compra. Talvez, a exemplo dos genéricos, os medicamentos de referência poderiam ser identificados por uma tarja ou uma letra.

A política de medicamentos genéricos, além de regular preços e garantir a eficácia e a segurança deles, de forma a manter e/ou a aumentar a confiança da população e dos profissionais da saúde, nos medicamentos genéricos, deve divulgar informações para que a população possa decidir corretamente. Confiança é um fator-chave e terá maior êxito quando pensarmos em formação e informação (clara e independente) para prescritores, farmacêuticos e população sobre os genéricos.

\section{Colaboradores}

CR Blatt e SC Trauthman foram responsáveis pela coordenação da pesquisa e redação final do artigo. EH Schmidt, S Marchesan, LM Silva e JL Martins participaram da elaboração do projeto, coleta de dados e redação inicial do artigo. 


\section{Referências}

1. Organização Mundial da Saúde (OMS). Perspectivas políticas sobre medicamentos da OMS. Genebra: Organização Mundial da Saúde (OMS); 2000.

2. Bermudez J. Medicamentos genéricos: uma alternativa para o mercado brasileiro. Cad Saude Publica 1994; 10(3):368-378.

3. Vieira FS, Zucchi P. Diferenças de preços entre medicamentos genéricos e de referência no Brasil. Rev Saude Publica 2006; 40(3):444-449.

4. Zara Yahni C, Segú Tolsa LS, Font Pous MF, Rovira J. La regulación de los medicamentos: teoría y práctica. Gaceta Sanitária 1998; 12:39-49.

5. Tobar F. Economía de los medicamentos genéricos en America Latina. Rev Panam Salud Publica 2008; 23(1):59-67.

6. Carvalho MCRD, Accioly Jr H, Raffin FN. Representações sociais do medicamento genérico por consumidores residentes em Natal, Rio Grande do Norte, Brasil. Cad Saude Publica 2006; 22(3):653-661.

7. Loyola MA. Medicamentos e saúde pública em tempos de AIDS: metamorfoses de uma política dependente. Cien Saude Colet 2008; 13(Supl.):763-778.

8. Quental C, Abreu, JC, Bomtempo JV, Gadelha, CAG. Medicamentos genéricos no Brasil: impactos das políticas públicas sobre a indústria nacional. Cien Saude Colet 2008; 13(Supl.):619-628.

9. Brasil. Lei No 9.787, de 10 de fevereiro de 1999. Altera a Lei no 6.360, de 23 de setembro de 1976, que dispõe sobre a vigilância sanitária, estabelece o medicamento genérico, dispõe sobre a utilização de nomes genéricos em produtos farmacêuticos e dá outras providências. Diário Oficial da União 1999; $11 \mathrm{fev}$.

10. Dias CRC, Romano-Lieber NS. Processo de implantação dos medicamentos genéricos no Brasil. Cad Saude Publica 2006;22(8):1661-1669.

11. Dias CRC. Medicamentos genéricos no Brasil de 1999 a 2002: análise da legislação, aspectos conjunturais e políticos [dissertação]. São Paulo: Faculdade de Saúde Pública; 2003.

12. Monteiro WM, Melo GC, Massunari GK, Hubner DV, Tasca RS. Avaliação da disponibilidade de medicamentos genéricos em farmácias e drogarias de Maringá (PR) e comparação de seus preços com os de referência e similares. Revista Brasileira de Ciências Farmacêuticas 2005; 41(3):333-343.

13. Jatene AD, Pina FAL, Caravante Junior FPG. Manual médico: medicamentos genéricos. São Paulo: Lemos; 2002.

14. Associação Brasileira das Indústrias de Medicamentos Genéricos. Mercado. [acessado $2008 \mathrm{dez} 15$ ]. Disponível em: http://progenericos.org.br/mercado. shtml.

15. Brasil. Ministério da Saúde (MS). Agência Nacional de Vigilância Sanitária (ANVISA). Pesquisa nacional de opinião pública sobre medicamentos genéricos. Brasília: Ministério da Saúde (MS); 2002.

16. Bertoldi A, Barros A, Hallal PC. Generic drugs in Brasil: known by many, used by few. Cad Saude Publica 2005; 21(6):1808-1815.

17. Cherobin J, Sandrini L. Nível de utilização de medicamentos genéricos no município de Tubarão. [Tcc]. Tubarão: Universidade do Sul de Santa Catarina; 2002
18. Costa EM, Medeiros FP. Medicamentos genéricos: grau de conhecimento e aceitação por indivíduos frequentadores de grupo da terceira idade no município de Tubarão-SC. [Tcc]. Tubarão: Universidade do Sul de Santa Catarina; 2006.

19. Faria MAS, Tavares-Neto J. Conhecimento popular sobre medicamento genérico em um distrito docente-assistencial do Município de Rio Branco, Estado do Acre, Brasil. Epidemiologia e Serviços de Saúde 2006; 15(3):37-45.

20. Rocha CE, Barros JAC, Silva MDP. Levantamento de dados sobre o conhecimento e informação acerca dos medicamentos genéricos em uma população de pacientes do serviço de saúde ambulatorial do Recife, Pernambuco, Brasil. Cad Saude Publica 2007; 23(5):1141-1150.

21. Le Pen C. L'economie des medicaments generiques. Journal d'Economie Medicale 1996; 14:413-437.

22. Garcia AJ, Martos F, Leiva F, Sánchez de La Cuesta F. Gac Sanit 2003; 17(2):144-149.

23. Rocha MAG, Martins RJ. Dispensação de medicamentos genéricos, conhecimentos, atitudes e práticas em farmácias comunitárias de Tubarão, SC. [Tcc]. Tubarão: Universidade do Sul de Santa Catarina; 2006.

24. Valles JA, Barreiro M, Cereza G, Ferro JJ, Martinez MJ, Cucurrull E, Barceló E. Aceptación de los fármacos genéricos en equipos de atención primaria: efecto de una intervención educativa y de los precios de referencia. Gac Sanit 2002; 16(6):505-510.

25. Brasil. Banco de dados do Sistema Único de Saúde (Datasus). Caderno de Informação em Saúde. [acessado 2007 jun 07]. Disponível em: http://tabnet. datasus.gov.br/tabdata/cadernos/sc.htm

26. Associação Brasileira de Empresas de Pesquisa (ABEP). Critério de Classificação Econômica Brasil: dados com base no levantamento sócio econômico 2000, IBOPE. 2003. [acessado 2007 nov 5]. Disponível em: http://www.abep.org/codigosguias/ABEP CCEB.pdf.

27. Associação Brasileira das Indústrias de Medicamentos Genéricos. Pro genéricos. [acessado 2008 nov 22]. Disponível em: http://www.progenericos.org. br/mercado.htm

28. Hernandez JMC, Júnior EFO. A troca de medicamentos no ponto de venda e o mercado de medicamentos genéricos. Revista de Negócios 2006; 11(3):4160.

29. Rumel D, Nishioka SA, Santos AAM. Intercambialidade de medicamentos: abordagem clínica e o ponto de vista do consumidor. Rev Saude Publica 2006; 40(5):921-927.

30. Oliete MB, Bouza CT, Bustillo BM, Cuesta TS, León MN. Opinion de los usuarios de atencion primaria sobre los medicamentos genericos y el coste de la medicacion. Aten Primaria 2003; 31(3):170-177.

31. Angell M. A verdade sobre os laboratórios farmacêuticos: como somos enganados e o que podemos fazer a respeito. 3a ed. Rio de Janeiro: Record; 2003.

Artigo apresentado em 26/07/2009

Aprovado em 04/10/2009

Versão final apresentada em 19/10/2009 